\title{
Immunological basis of early clearance of Mycobacterium tuberculosis infection: the role of natural killer cells
}

\author{
Fekadu Abebe ${ }^{1}$ \\ ${ }^{1}$ University of Oslo Faculty of Medicine
}

September 28, 2020

\begin{abstract}
Tuberculosis kills more people than any other single infectious disease globally. Despite decades of research, there is no vaccine to prevent TB transmission. Bacille Calmette-Guerin (BCG) vaccine developed a century ago has little effect on pulmonary TB and does not control transmission. Lack of an effective vaccine emanates from lack of knowledge on correlates of protective immunity on which to base vaccine design and development. However, some household contacts who are extensively exposed to Mtb infection remain persistently negative to tuberculin skin test and interferon-gamma assay. These individuals called "resisters" clear Mtb infection early before the development of acquired immunity. The immunological basis of early Mtb clearance is yet to be established, however, innate lymphocytes such as monocytes/macrophages, dendritic cells, neutrophils and natural killer cells, and innate like $\mathrm{T}$ cells such as mucosal associated invariant $\mathrm{T}$ cells, invariant natural killer $\mathrm{T}$ cells and gamma-delta $(\gamma \delta) \mathrm{T}$ cells have been implicated in this early protection. One of the cells that has attracted increasing attention in recent years, in protection against Mtb is the natural killer cell. Emerging data from animal and epidemiological studies indicate that NK cells may play a significant role in the fight against Mtb. NK cells express various surface markers to recognize and kill both Mtb and Mtb-infected cells. In this review, recent advances in our understanding of NK cells in the fight against Mtb early during infection, with emphasis on cohort studies, will be presented.
\end{abstract}

\section{Hosted file}

NK cell manuscript-CEI formatted.pdf available at https://authorea.com/users/362469/articles/ 483617-immunological-basis-of-early-clearance-of-mycobacterium-tuberculosis-infectionthe-role-of-natural-killer-cells 
Fig. 1

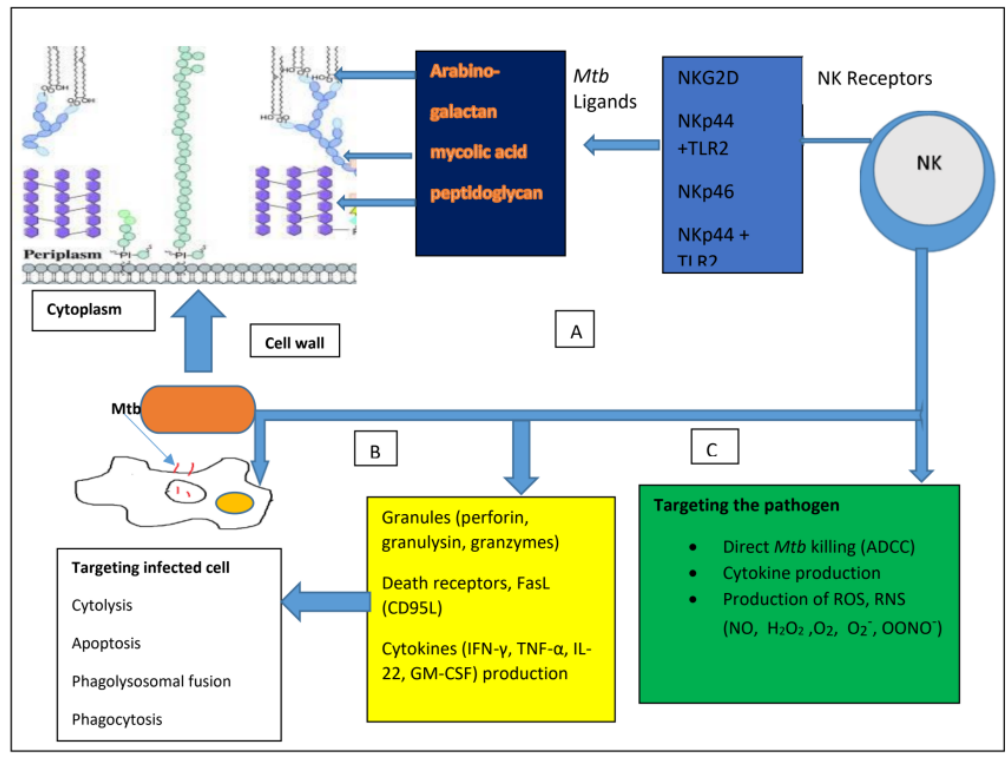

